\title{
Evaluation of Elite Wheat Germ Plasm for Resistance to Tan Spot
}

P. K. Singh and M. Mergoum, Department of Plant Sciences, 166-Loftsgard Hall, North Dakota State University, Fargo 58105; S. Ali and T. B. Adhikari, Department of Plant Pathology, 306-Walster Hall, North Dakota State University, Fargo 58105; E. M. Elias, Department of Plant Sciences, 166-Loftsgard Hall, North Dakota State University, Fargo 58105; J. A. Anderson, Department of Agronomy and Plant Genetics, 411-Borlaug Hall, 1991 Upper Buford Circle, University of Minnesota, St. Paul 55108; K. D. Glover, Plant Science Department, NPB 247 Box 2140-C, South Dakota State University, Brookings 57007; and W. A. Berzonsky, Department of Plant Sciences, 166Loftsgard Hall, North Dakota State University, Fargo 58105

\begin{abstract}
Singh, P. K., Mergoum, M., Ali, S., Adhikari, T. B., Elias, E. M., Anderson, J. A., Glover, K. D., and Berzonsky, W. A. 2006. Evaluation of elite wheat germ plasm for resistance to tan spot. Plant Dis. 90:1320-1325.

Tan spot, caused by Pyrenophora tritici-repentis, is a serious foliar disease of wheat (Triticum aestivum) in North America. Control of tan spot through management practices and fungicide application is possible; however, the use of resistant varieties is the most effective and economical means of controlling tan spot. This study was conducted to determine the disease reaction of 126 elite hard red spring, white, and durum wheat varieties and advanced breeding lines collected from the northern Great Plains of the United States and Canada to individual races/toxins of $P$. tritici-repentis. Seedling evaluation of the 126 genotypes was done under controlled environmental conditions with virulent races 2, 3, and 5 of $P$. tritici-repentis and toxins Ptr ToxA and Ptr ToxB. Based on disease reactions, two resistant varieties and two advanced breeding lines adapted to the northern Great Plains were found to be resistant to all the races and insensitive to the toxins tested. Additionally, six genetically diverse lines/varieties were identified to be resistant to tan spot; however, these sources may not be well adapted to the northern Great Plains. These results suggest that the wheat germ plasm contains a broad genetic base for resistance to the most prevalent races of $P$. tritici-repentis in North America, and the resistant sources identified in this study may be utilized in wheat breeding programs to develop tan spot resistant varieties.
\end{abstract}

The fungus Pyrenophora tritici-repentis (Died.) Drechs. (anamorph: Drechslera tritici-repentis (Died.) Shoem.) causes tan spot of wheat (Triticum aestivum L.), an important foliar disease affecting grain quality and production $(6,26)$. Intensified wheat production, changes in cultural practices including shifts from conventional tillage and stubble burning to reduced tillage practices, and shorter crop rotations have resulted in development of tan spot in epidemic proportions worldwide (7). Management practices such as use of nonhost plants in the crop rotation, destruction and avoidance of infested straw, stubble, and volunteer plants by either burning or burying are useful for controlling tan spot. However, stubble burning and tillage increase the risk of soil erosion and can contribute to pollution of the environment. Similarly, although the application of fungicides is effective in controlling tan spot,

Corresponding author: M. Mergoum

E-mail: Mohamed.Mergoum@ndsu.edu

Accepted for publication 7 June 2006.

DOI: 10.1094/PD-90-1320

(C) 2006 The American Phytopathological Society their use may leave undesirable residues and is not cost-effective due to low grain prices. Therefore, resistant varieties are the most effective and economical means of controlling tan spot of wheat (7).

$P$. tritici-repentis induces two distinct symptoms on susceptible wheat cultivars, tan necrosis and extensive chlorosis, both of which are under independent genetic control in the host $(19,24)$. Based on the ability to induce necrosis and/or chlorosis on a set of wheat differential cultivars, isolates of $P$. tritici-repentis are presently classified into eight races (26). In recent years, preliminary reports of occurrence of three more virulent types of $P$. triticirepentis have been made $(1,3,27)$. Races 1 to 5 of $P$. tritici-repentis have been reported to occur in North America $(2,22)$, while races 6,7 , and 8 have been identified from North Africa and the Middle East region (26).

The tan spot fungus produces different host-specific toxins, which have been associated with the necrosis and chlorosis symptoms induced on susceptible wheat cultivars. One well-characterized hostselective proteinaceous toxin, Ptr ToxA $(6,22)$, produced by races $1,2,7$, and 8 (26), is the main factor causing the necrotic symptom in susceptible wheat culti- vars. Two chlorosis-inducing host-specific toxins, designated as Ptr ToxB (29), isolated from race 5, and Ptr ToxC (10), isolated from race 1 , have been identified and well characterized. Genetic studies have established that sensitivity to toxins Ptr ToxA, Ptr ToxB, and Ptr ToxC and susceptibility to their producer races are each controlled by the same gene $(10,18,29)$. They also observed that toxins could be used in screening for resistance as a surrogate for spore inoculation.

Resistance to tan spot of wheat is quantitatively $(11,14)$ or qualitatively $(8,9,19$, 24,33) inherited. Genetic studies indicate that a single recessive gene, $t s n 1$, controls resistance to necrosis caused by races 1 and 2 in both durum and common wheat and is located on the long arm of chromosome 5B (4,13). Effertz et al. (10) mapped the locus controlling insensitivity to toxin Ptr ToxC to the short arm of chromosome $1 \mathrm{~A}$ in common wheat. Friesen and Faris (16) identified in common wheat a major quantitative trait locus (QTL) associated with insensitivity to Ptr ToxB to the distal tip of the short arm of chromosome 2B, and this QTL accounted for $69 \%$ of the phenotypic variation.

The epidemic occurrence of tan spot in recent years in major wheat-growing regions worldwide and high pathogenic variability in populations of $P$. tritici-repentis have resulted in a greater urgency to incorporate multiple resistance genes and broaden the genetic base of resistance in adapted varieties $(7,18,28,34)$. Breeding for resistance to tan spot may be enhanced if additional resistance sources that are effective against multiple races of $P$. triticirepentis are identified. The main objectives of this study were to (i) screen elite wheat breeding lines and currently released wheat varieties to the major virulence present in P. tritici-repentis in North America, (ii) identify resistance sources effective against tan spot, and (iii) provide wheat breeders with sources of resistance and effective means of evaluating tan spot resistance to allow them to transfer tan spot resistance into adapted wheat.

\section{MATERIALS AND METHODS}

Plant material. A collection of 126 elite wheat germ plasm including hard red 
spring wheat, white wheat, and durum varieties along with advanced breeding materials from both private and public wheat breeding programs of the northern Great Plains of the United States and Canada were evaluated in greenhouse studies. The different types of wheat genotypes used in this study, their pedigrees, and sources of origin are given in Table 1.

Spore inoculation. A single-spore culture of isolates Ptr 86-124 (race 2), Ptr 331-9 (race 3), and Ptr DW13 (race 5) was used to produce spore inoculum. The isolates Ptr 86-124 and Ptr 331-9 were provided to North Dakota State University wheat leaf diseases laboratory by L. Lamari, University of Manitoba, Winnipeg, Canada. The race designation of each isolate was confirmed in our laboratory. Spore inoculum was produced by the method of Lamari and Bernier (21). Throughout this study, the spore inoculum concentration was 4,000 conidia per milliliter.

Seed of the 126 wheat genotypes, including checks Glenlea, Saloumini, 6B365 , and $6 \mathrm{~B}-662$, was pregerminated to minimize the chances of growth variation prior to planting in plastic cones $(3.8 \mathrm{~cm}$ in diameter and $20 \mathrm{~cm}$ long) filled with Fison sunshine blend \#1 (Fison Horticulture, Vancouver, B.C.). The seedlings were grown in the greenhouse at an average temperature of $21^{\circ} \mathrm{C}$ with a $16-\mathrm{h}$ photoperiod. Four seeds were planted in each cone. The seedlings were watered and fertilized as needed. Plants at the two-leaf stage were sprayed with the conidial suspension until runoff using a $\mathrm{CO}_{2}$ pressurized hand sprayer. Following inoculation, the seedlings were incubated for $24 \mathrm{~h}$ under continuous leaf wetness in a mist chamber and then placed on benches in a growth chamber set at $22 / 17^{\circ} \mathrm{C}$ (day/ night) temperature with a $16-\mathrm{h}$ photoperiod.

Eight days after spore inoculation, the seedlings were rated for their disease reaction, using a 1 to 5 lesion-type rating scale developed by Lamari and Bernier (21). Necrotic or chlorotic symptoms observed on genotypes were recorded.

Toxin and culture filtrate infiltration. The toxin Ptr ToxA, produced from isolate Ptr 86-124 (race 2), was kindly provided by S. W. Meinhardt, Department of Plant Pathology, North Dakota State University, Fargo. The toxin was purified as described previously by Zhang et al. (36). Culture filtrate containing the toxin Ptr ToxB was produced from isolate Ptr DW13 (race 5) by the procedures of Orolaza et al. (29).

Approximately $25 \mu \mathrm{l}$ of the toxin/culture filtrate was infiltrated into the wheat leaves using a 1-ml syringe without a needle. The infiltrated second leaf was marked by a nontoxic permanent marker. Plant reactions to the toxin/culture filtrate infiltration were recorded 4 days after infiltration. The presence or absence of necrosis/chlorosis at the site of infiltration indicated sensitiv- ity or insensitivity to the culture filtrate, respectively.

Experimental design and statistical analysis. To evaluate the 126 wheat germ plasm for each of the three races of $P$. tritici-repentis, two experiments were conducted in the greenhouse in spring and fall of 2005 for each race. Each experiment was conducted as a randomized block design with three replicates. Each replicate consisted of the 126 wheat genotypes planted in cones. Four plants planted in a cone formed the experimental unit.

An analysis of variance (ANOVA) for each race causing tan spot was performed using the Statistical Analysis System version 8.2 (31). The existence of significant differences $(P<0.05)$ in disease reaction among genotypes was calculated with PROC GLM using LSD option. For the reaction of 126 genotypes to the toxin/culture filtrate, four plants per genotype were infiltrated with the toxin Ptr ToxA and culture filtrate produced by race 5 (Ptr ToxB).

\section{RESULTS AND DISCUSSION}

Analysis of variance of the disease data revealed significant $(P<0.05)$ differences among genotypes in all experiments for the three races of $P$. tritici-repentis. There was no significant $(P<0.05)$ genotype $\times$ experiment interaction for any of the races studied. The results of statistical analysis permitted combining the two experiments for each of the three races, and mean disease score of each genotype with respect to races 2,3 , and 5 and the respective LSD $\left(P_{0.05}\right)$ for each race is given in Table 1.

Ten genotypes showed resistance to all races and insensitivity to all toxins of $P$. tritici-repentis tested (Table 1). Among these resistant genotypes, two recently released varieties, Knudson and Oklee, and two advanced breeding lines, N99-0241 and MN03291, are well adapted to the northern Great Plains of the United States and, hence, can be successfully used in various wheat breeding programs. In order to broaden the genetic base of resistance to tan spot, the resistance from lines 92 MREHTR28B, CIMMYT L \#18, and Intros \#7 and varieties Salamouni and Erik can also be used. These sources of resistance may not be well adapted to the Great Plains of North America but can be utilized for broadening the genetic base of resistant germ plasm. All durum lines screened were susceptible; however, a tetraploid accession, T. turgidum \#283 (PI PI352519), exhibited high resistance to all races and insensitivity to all toxins of $P$. tritici-repentis tested. The pedigree of resistant sources identified in this study indicate that they come from diverse genetic background and may possibly possess different resistance genes that can be utilized in gene pyramiding and developing cultivars with broad and durable resistance to tan spot.
The majority of genotypes evaluated with races 2 and 5 and their respective toxins gave similar reactions (Table 1). Similar findings were observed by other researchers $(9,19)$. However, a few genotypes gave differential response to races 2 and 5 spore inoculation and their toxins. Differential responses to toxin and spore inoculation have also been observed by others $(17,30)$. Friesen et al. (15) observed that segregation of insensitivity to necrosis induced by toxin Ptr ToxA was controlled by a single gene, while the disease reaction of the recombinant inbred lines to spore inoculation by race 2 revealed that four to five genes may control resistance in the population. In the lines showing differential response, they observed that lines were insensitive to Ptr ToxA but susceptible to spore suspension and not vice versa. Similar results were observed in this study. Additional experiments need to be conducted to reveal the exact reasons for the differential response of the genotypes identified in this study.

The time required for germ plasm evaluation of tan spot reaction by toxin/culture filtrate infiltration (4 days) is half the duration required by spore suspension ( 8 days). The toxins/culture filtrate can be stored for a very long time and can be reused in different batches. We estimate the expense involved in disease testing using toxin/culture filtrate would be less compared with that for spore inoculation in wheat breeding programs. Reaction to the culture filtrate is clear and easily differentiates between sensitive and insensitive plants (19). Toxin/culture filtrate infiltration is less influenced by environmental conditions (35) and can easily be combined with screening for resistance to other leaf-spotting diseases (personal observation). These results indicate that toxins/culture filtrates can be used for largescale evaluation of early generation breeding material, but final confirmation of tan spot resistance has to be done by spore inoculum to avoid selection of lines that are resistant to toxins but susceptible to spore inoculum.

Tan spot has become a major component of the leaf spotting disease complex of wheat in the northern Great Plains of the United States and Canada $(20,28)$. Both private and public wheat-breeding programs have given major emphasis to breeding for resistance to tan spot. Screening for tan spot resistance under natural infection is not always considered reliable enough for field screening (5). Artificial infection can be obtained by spreading infected straw or by spraying spore inoculum and covering with plastic tents to maintain humidity (23). However, these methods have practical constraints like production and quantification of large amounts of inoculum, maintenance of high humidity, and disease assessment based on percentage of diseased leaf area. As a re- 
Table 1. Wheat type, origin, pedigree, and disease reaction of 126 elite wheat genotypes to Pyrenophora tritici-repentis races 2,3 , and 5, and toxins Ptr ToxA and Ptr ToxB

\begin{tabular}{|c|c|c|c|c|c|c|c|}
\hline \multirow[b]{2}{*}{ Genotype } & \multirow[b]{2}{*}{ Type $^{\mathrm{a}}$-origin ${ }^{\mathrm{b}}$} & \multirow[b]{2}{*}{ Pedigree } & \multicolumn{3}{|c|}{ Race $^{\mathrm{c}}$} & \multicolumn{2}{|c|}{ Toxin $^{\mathrm{d}}$} \\
\hline & & & $\mathbf{R}-2$ & $\mathbf{R - 3}$ & R-5 & ToxA & ToxB \\
\hline Oxen & HRSW-SD & SDY366A/SDZ004A & $3.5 \mathrm{~N}$ & $1.8 \mathrm{R}$ & $1.9 \mathrm{R}$ & + & - \\
\hline Ingot & HRSW-SD & Butte86/SD3004/Dalen & $3.5 \mathrm{~N}$ & $2.3 \mathrm{C}$ & $2.8 \mathrm{C}$ & + & - \\
\hline Norpro & HRSW-CO & N93-0119 & $1.9 \mathrm{R}$ & $1.7 \mathrm{R}$ & $1.4 \mathrm{R}$ & + & - \\
\hline Parshall & HRSW-ND & Keene//Grandin*2/Glupro & $3.1 \mathrm{~N}$ & $1.6 \mathrm{R}$ & $3.8 \mathrm{C}$ & + & + \\
\hline Reeder & HRSW-ND & IAS20*4/H567.71//Stoa/3/ND 674 & $2.5 \mathrm{~N}$ & $1.5 \mathrm{R}$ & $3.9 \mathrm{C}$ & + & + \\
\hline Alsen & HRSW-ND & ND 716 (Sumai3/Wheaton//Grandin) & $3.5 \mathrm{~N}$ & $1.9 \mathrm{R}$ & $2.0 \mathrm{R}$ & + & - \\
\hline Knudson & HRSW-CO & Karl/Krona/3/Bergen//Erik/MN73167 & $1.6 \mathrm{R}$ & $1.6 \mathrm{R}$ & $1.8 \mathrm{R}$ & - & - \\
\hline Hanna & HRSW-CO & N93-2424/AC DOMAIN & $3.0 \mathrm{~N}$ & $3.1 \mathrm{C}$ & $3.8 \mathrm{C}$ & + & + \\
\hline Briggs & HRSW-SD & BW114/Bergen/SD3097 & $2.2 \mathrm{~N}$ & $2.4 \mathrm{C}$ & $2.5 \mathrm{C}$ & + & + \\
\hline Granite & HRSW-MT & Unknown & $1.9 \mathrm{R}$ & $2.2 \mathrm{C}$ & $2.6 \mathrm{C}$ & - & - \\
\hline Oklee & HRSW-MN & 2375/SBF0670 & $1.5 \mathrm{R}$ & $1.4 \mathrm{R}$ & $1.3 \mathrm{R}$ & - & - \\
\hline Dapps & HRSW-ND & Kitt/Amidon//GR/Stoa sib & $2.1 \mathrm{~N}$ & $2.8 \mathrm{C}$ & $4.0 \mathrm{C}$ & - & + \\
\hline Steele-ND & HRSW-ND & Parshall/ND706 & $3.7 \mathrm{~N}$ & $3.0 \mathrm{C}$ & $4.2 \mathrm{C}$ & + & + \\
\hline Fryer & HRSW-CO & Sonja/Vance//Sumai3/Dalen & $2.2 \mathrm{~N}$ & $1.6 \mathrm{R}$ & $2.0 \mathrm{R}$ & - & - \\
\hline Trooper & HRSW-MT & Keystone/Ivan & $2.0 \mathrm{R}$ & $2.0 \mathrm{R}$ & $1.6 \mathrm{R}$ & - & - \\
\hline Banton & HRSW-MN & Buck Antorcha/HJ 9 & $3.1 \mathrm{~N}$ & $3.4 \mathrm{C}$ & $3.9 \mathrm{C}$ & + & + \\
\hline Saturn & HRSW-MN & ANB'S//HEGE312-75-262/CHAT'S & $2.9 \mathrm{~N}$ & $2.1 \mathrm{C}$ & $1.6 \mathrm{R}$ & - & - \\
\hline Polaris & HRSW-MN & Lona/4/Kent/B564//SO/3/Kent/2*B564//SAP & $2.2 \mathrm{~N}$ & $1.6 \mathrm{R}$ & $2.6 \mathrm{C}$ & - & - \\
\hline 2375 & HRSW-ND & Olaf//Era/Suqamuxi68/3/Cis/ND487//Lark & $2.8 \mathrm{~N}$ & $2.4 \mathrm{C}$ & $1.9 \mathrm{R}$ & + & - \\
\hline Verde & HRSW-MN & MN7663/SBY35A & $2.5 \mathrm{~N}$ & $2.0 \mathrm{R}$ & $2.8 \mathrm{C}$ & - & + \\
\hline Keene & HRSW-ND & Stoa's'/3/IAS20*4//H567.71//Amidon & $3.6 \mathrm{~N}$ & $1.9 \mathrm{R}$ & $4.3 \mathrm{C}$ & - & + \\
\hline Butte 86 & HRSW-ND & Butte*2/3/ND551//Butte*2/ND507 & $3.5 \mathrm{~N}$ & $1.9 \mathrm{R}$ & $4.2 \mathrm{C}$ & + & + \\
\hline McNeal & HRSW-MT & PI125000/Centana//PK176/Frontiera/3/Glenman & $2.9 \mathrm{~N}$ & $1.3 \mathrm{R}$ & $1.6 \mathrm{R}$ & - & - \\
\hline Walworth & HRSW-SD & SD 3116/OXEN & $3.1 \mathrm{~N}$ & $1.7 \mathrm{R}$ & $1.9 \mathrm{R}$ & + & - \\
\hline Dandy & HRSW-MN & Unknown & $3.4 \mathrm{~N}$ & $2.0 \mathrm{R}$ & $2.0 \mathrm{R}$ & + & - \\
\hline Granger & HRSW-SD & SD8070/SD3165 & $2.9 \mathrm{~N}$ & $2.1 \mathrm{C}$ & $2.6 \mathrm{C}$ & + & + \\
\hline SD3618 & HRSW-SD & SD8119/SD3236 & $3.6 \mathrm{~N}$ & $3.1 \mathrm{C}$ & $3.2 \mathrm{C}$ & + & + \\
\hline SD3635 & HRSW-SD & SD8070/SD3118//SD3225 & $3.6 \mathrm{~N}$ & $3.1 \mathrm{C}$ & $3.1 \mathrm{C}$ & + & + \\
\hline SD3668 & HRSW-SD & SD8089/SD8070 & $3.2 \mathrm{~N}$ & $1.8 \mathrm{R}$ & $2.1 \mathrm{C}$ & + & + \\
\hline SD3687 & HRSW-SD & SD3305/KS91W005-1-4//SD8089 & $3.4 \mathrm{~N}$ & $2.3 \mathrm{C}$ & $1.9 \mathrm{R}$ & + & - \\
\hline SD3746 & HRSW-SD & SD93380/SD3335//Oxen & $3.5 \mathrm{~N}$ & $3.1 \mathrm{C}$ & $3.9 \mathrm{C}$ & + & + \\
\hline SD3827 & HRSW-SD & W94-320/SD3367//SD3335 & $3.6 \mathrm{~N}$ & $2.6 \mathrm{C}$ & $2.8 \mathrm{C}$ & + & - \\
\hline SD3851 & HRSW-SD & ND 2897/SD3219//SD3414 & $2.6 \mathrm{~N}$ & $2.5 \mathrm{C}$ & $2.5 \mathrm{C}$ & + & + \\
\hline SD3854 & HRSW-SD & ND 2897/SD3219//SD3414 & $3.3 \mathrm{~N}$ & $2.0 \mathrm{R}$ & $4.1 \mathrm{C}$ & + & + \\
\hline SD3860 & HRSW-SD & AZ99FHB-RC & $3.2 \mathrm{~N}$ & $2.1 \mathrm{C}$ & $2.2 \mathrm{C}$ & - & - \\
\hline SD3868 & HRSW-SD & ND 2897/SD3479//SD3411 & $3.3 \mathrm{~N}$ & $1.8 \mathrm{R}$ & $2.5 \mathrm{C}$ & + & + \\
\hline SD3870 & HRSW-SD & FHBCOMP & $3.1 \mathrm{~N}$ & $1.8 \mathrm{R}$ & $3.9 \mathrm{C}$ & + & + \\
\hline BZ 998-447W & WW-MT & Spillman/WestBred 906R & $3.0 \mathrm{~N}$ & $2.0 \mathrm{R}$ & $2.9 \mathrm{C}$ & - & + \\
\hline CA-902-701 & OW-MT & Keystone/Granite & $2.1 \mathrm{~N}$ & $2.8 \mathrm{C}$ & $1.8 \mathrm{R}$ & - & - \\
\hline CA-901-580W & WW-MT & White Sharpshooter/Lars & $3.0 \mathrm{~N}$ & $1.8 \mathrm{R}$ & $1.8 \mathrm{R}$ & - & - \\
\hline N99-0241 & HRSW-CO & N93-0090/Hamer & $1.9 \mathrm{R}$ & $1.4 \mathrm{R}$ & $1.8 \mathrm{R}$ & - & - \\
\hline 96S0404-03 & HRSW-CO & Hamer//RHT21/Lars & $1.9 \mathrm{R}$ & $1.5 \mathrm{R}$ & $2.5 \mathrm{C}$ & - & + \\
\hline 98S0051-1 & HRSW-CO & N93-0243//CHISCAB\#7/N93-0338 & $2.1 \mathrm{~N}$ & $1.3 \mathrm{R}$ & $3.6 \mathrm{C}$ & + & + \\
\hline 98S0127-06 & HRSW-CO & N97-0214/3/N93-0338//Sumai3/Dalen & $2.5 \mathrm{~N}$ & $1.8 \mathrm{R}$ & $2.1 \mathrm{C}$ & + & + \\
\hline 97S0254-8-1 & HRSW-CO & N94-0236/IVAN//Verde & $2.0 \mathrm{R}$ & $2.5 \mathrm{C}$ & $2.2 \mathrm{C}$ & - & - \\
\hline MN97369 & HRSW-MN & MN97369-98*4/Pavon & $3.0 \mathrm{~N}$ & $1.5 \mathrm{R}$ & $3.8 \mathrm{C}$ & + & + \\
\hline MN97448 & HRSW-MN & HJ98/SHARP & $3.3 \mathrm{~N}$ & $1.8 \mathrm{R}$ & $2.2 \mathrm{C}$ & + & + \\
\hline MN97695 & HRSW-MN & MN92387/SBE0303-23 & $3.2 \mathrm{~N}$ & $1.9 \mathrm{R}$ & $4.0 \mathrm{C}$ & + & + \\
\hline MN99017 & HRSW-MN & Fujian 5114/Grandin & $2.5 \mathrm{~N}$ & $2.5 \mathrm{C}$ & $4.3 \mathrm{C}$ & + & + \\
\hline MN99436-6 & HRSW-MN & Norlander/HJ98 & $3.4 \mathrm{~N}$ & $1.7 \mathrm{R}$ & $1.7 \mathrm{R}$ & + & - \\
\hline MN00232-2-2 & HRSW-MN & MN2540W/KULM//ND2710 & $3.0 \mathrm{~N}$ & $2.2 \mathrm{C}$ & $3.9 \mathrm{C}$ & + & + \\
\hline MN00258-2 & HRSW-MN & INGOT/HJ98 & $3.2 \mathrm{~N}$ & $2.3 \mathrm{C}$ & $1.9 \mathrm{R}$ & + & - \\
\hline MN00261-4 & HRSW-MN & MN95286/MN94155//Verde & $2.3 \mathrm{~N}$ & $1.8 \mathrm{R}$ & $1.6 \mathrm{R}$ & + & - \\
\hline MN01261-8 & HRSW-MN & MN95229/ND694 & $2.9 \mathrm{~N}$ & $1.8 \mathrm{R}$ & $3.8 \mathrm{C}$ & - & + \\
\hline MN01281 & HRSW-MN & SD-AD B9/MN93434(2375/Grandin) & $3.1 \mathrm{~N}$ & $1.8 \mathrm{R}$ & $2.4 \mathrm{C}$ & + & + \\
\hline MN01333-A & HRSW-MN & MN97008/MN97695 & $3.1 \mathrm{~N}$ & $1.7 \mathrm{R}$ & $2.0 \mathrm{R}$ & + & - \\
\hline MN01108-6 & HRSW-MN & MN95229//MF9606/Aberdeen 9 & $3.4 \mathrm{~N}$ & $2.7 \mathrm{C}$ & $3.7 \mathrm{C}$ & + & + \\
\hline MN01116 & HRSW-MN & $\begin{array}{l}\text { MN95211(PAR11(MRNC/BUC//BLD/PSN)/MN90124)// } \\
\text { LizCimmyt9/MN95023 }\end{array}$ & $3.4 \mathrm{~N}$ & $1.8 \mathrm{R}$ & $3.8 \mathrm{C}$ & + & + \\
\hline \multirow[t]{2}{*}{ MN01180-4 } & HRSW-MN & LizCimmyt17/Parshall//HJ98 & $2.4 \mathrm{~N}$ & $1.5 \mathrm{R}$ & $1.5 \mathrm{R}$ & - & - \\
\hline & & & & & \multicolumn{3}{|c|}{ (continued on next page) } \\
\hline
\end{tabular}

a The different types of wheat: HRSW, hard red spring wheat; OW, other wheat; and WW, white wheat.

${ }^{\mathrm{b}}$ Origins of the genotypes studied: CAN, Canada; CO, Colorado; KS, Kansas; MEX, Mexico; MN, Minnesota; MT, Montana; ND, North Dakota; SD, South Dakota; and WA, Washington.

${ }^{\mathrm{c}}$ Plants were rated on a 1 to 5 lesion type rating scale (23) with 1 to 2 considered resistant (R) and 3 to 5 considered susceptible with necrosis (N) or chlorosis (C) symptoms.

${ }^{\mathrm{d}}$ Reactions to toxin/culture filtrate were scored as + and - , indicating sensitivity and insensitivity, respectively. Toxin Ptr ToxA is produced by race 2 and induces necrosis, while Ptr ToxB is produced by race 5 and causes chlorosis. 
Table 1. (continued from previous page)

\begin{tabular}{|c|c|c|c|c|c|c|c|}
\hline \multirow[b]{2}{*}{ Genotype } & \multirow[b]{2}{*}{ Type $^{\mathrm{a}}$-origin ${ }^{\mathrm{b}}$} & \multirow[b]{2}{*}{ Pedigree } & \multicolumn{3}{|c|}{ Races $^{c}$} & \multicolumn{2}{|c|}{ Toxins $^{d}$} \\
\hline & & & $\mathbf{R}-2$ & R-3 & R-5 & ToxA & ToxB \\
\hline MN01278-7 & HRSW-MN & MN94200/SD-AD B61 & $3.7 \mathrm{~N}$ & $1.8 \mathrm{R}$ & $1.8 \mathrm{R}$ & + & - \\
\hline MN02255 & HRSW-MN & ND721/MN97448 & $3.5 \mathrm{~N}$ & $1.9 \mathrm{R}$ & $3.9 \mathrm{C}$ & - & + \\
\hline MN02258 & HRSW-MN & ND724/MN97448 & $2.5 \mathrm{~N}$ & $1.9 \mathrm{R}$ & $2.3 \mathrm{C}$ & + & - \\
\hline MN02296-A & HRSW-MN & Verde/MN93415//HJ98 & $2.0 \mathrm{R}$ & $1.5 \mathrm{R}$ & $3.4 \mathrm{C}$ & + & + \\
\hline MN03098 & HRSW-MN & MN97803-10/DANDY-1 & $2.2 \mathrm{~N}$ & $3.0 \mathrm{C}$ & $1.8 \mathrm{R}$ & - & - \\
\hline MN03119 & HRSW-MN & Alsen-1/MN97803-10 & $3.2 \mathrm{~N}$ & $2.0 \mathrm{R}$ & $1.7 \mathrm{R}$ & - & - \\
\hline MN03130 & HRSW-MN & MN97695-4/MN97448-17 & $3.2 \mathrm{~N}$ & $2.0 \mathrm{R}$ & $1.6 \mathrm{R}$ & + & - \\
\hline MN03148 & HRSW-MN & MN99390-1/MN97803-10 & $2.8 \mathrm{~N}$ & $1.8 \mathrm{R}$ & $3.6 \mathrm{C}$ & - & + \\
\hline MN03239 & HRSW-MN & MN98543/MN97448 & $2.8 \mathrm{~N}$ & $1.9 \mathrm{R}$ & $3.9 \mathrm{C}$ & - & + \\
\hline MN03243 & HRSW-MN & 01X019-3/MN97803-10 & $2.6 \mathrm{~N}$ & $3.0 \mathrm{C}$ & $4.0 \mathrm{C}$ & + & + \\
\hline MN03279 & HRSW-MN & MN98224/MN97448 & $2.1 \mathrm{~N}$ & $1.7 \mathrm{R}$ & $1.6 \mathrm{R}$ & - & - \\
\hline MN03291 & HRSW-MN & MN98389/MN98021 & $2.0 \mathrm{R}$ & $1.4 \mathrm{R}$ & $1.9 \mathrm{R}$ & - & - \\
\hline MN03308 & HRSW-MN & MN95002//Parshall/MN97518 & $1.6 \mathrm{R}$ & $1.3 \mathrm{R}$ & $3.5 \mathrm{C}$ & - & + \\
\hline MN03328 & HRSW-MN & Alsen-1/MN97448-17 & $2.7 \mathrm{~N}$ & $1.9 \mathrm{R}$ & $2.5 \mathrm{C}$ & + & + \\
\hline WA007925 & OW-WA & Spillman/WPB906R//Sunstar II & $3.1 \mathrm{~N}$ & $2.1 \mathrm{C}$ & $1.7 \mathrm{R}$ & - & - \\
\hline WA007931 & WW-WA & PI591045/3/Tanager 'S'/Torim 73//Spillman & $3.7 \mathrm{~N}$ & $2.3 \mathrm{C}$ & $1.6 \mathrm{R}$ & + & - \\
\hline MT 0245 & HRSW-MT & MT9433/Reeder & $3.1 \mathrm{~N}$ & $2.0 \mathrm{R}$ & $2.0 \mathrm{R}$ & - & - \\
\hline MT 0249 & HRSW-MT & Reeder/MT9433 & $3.2 \mathrm{~N}$ & $1.9 \mathrm{R}$ & $4.0 \mathrm{C}$ & - & + \\
\hline ND 747 & HRSW-ND & ND2831//Parshall/ND706 & $3.9 \mathrm{~N}$ & $1.9 \mathrm{R}$ & $3.9 \mathrm{C}$ & + & + \\
\hline ND 751 & HRSW-ND & ND2709/3/GR*3//Ramsey/ND622/4/ND688/ND674 & $2.7 \mathrm{~N}$ & $1.7 \mathrm{R}$ & $1.7 \mathrm{R}$ & - & - \\
\hline ND 752 & HRSW-ND & ND2891/ND721 & $3.0 \mathrm{~N}$ & $2.0 \mathrm{R}$ & $4.3 \mathrm{C}$ & - & + \\
\hline ND 753 & HRSW-ND & ND721/Parshall & $3.0 \mathrm{~N}$ & $1.8 \mathrm{R}$ & $4.0 \mathrm{C}$ & - & + \\
\hline ND 755 & HRSW-ND & ND2849/ND721 & $3.2 \mathrm{~N}$ & $1.7 \mathrm{R}$ & $4.2 \mathrm{C}$ & + & + \\
\hline ND 756 & HRSW-ND & ND2849/ND721 & $2.3 \mathrm{~N}$ & $1.7 \mathrm{R}$ & $4.4 \mathrm{C}$ & - & + \\
\hline ND 800 & HRSW-ND & ND741 SELN (Parshall/ND706) & $3.3 \mathrm{~N}$ & $2.1 \mathrm{C}$ & $4.3 \mathrm{C}$ & + & + \\
\hline ND 801 & HRSW-ND & ND2849/ND721 & $2.3 \mathrm{~N}$ & $1.5 \mathrm{R}$ & $4.0 \mathrm{C}$ & _- & + \\
\hline ND 802 & HRSW-ND & ND721/ND725 & $3.5 \mathrm{~N}$ & $1.8 \mathrm{R}$ & $2.3 \mathrm{C}$ & - & + \\
\hline ND 803 & HRSW-ND & ND2831/FO.2808 & $2.4 \mathrm{~N}$ & $1.5 \mathrm{R}$ & $3.6 \mathrm{C}$ & - & + \\
\hline ND 804 & HRSW-ND & ND744 SELN & $3.1 \mathrm{~N}$ & $1.7 \mathrm{R}$ & $2.1 \mathrm{C}$ & + & + \\
\hline ND 805 & HRSW-ND & ND2857/3/KITT/Amidon//GR/Stoa SIB & $1.7 \mathrm{R}$ & $2.1 \mathrm{C}$ & $3.7 \mathrm{C}$ & - & + \\
\hline ND 806 & HRSW-ND & SD3348/Reeder//ND721 & $2.7 \mathrm{~N}$ & $1.6 \mathrm{R}$ & $3.5 \mathrm{C}$ & - & + \\
\hline ND 807 & HRSW-ND & ND2831//Parshall/ND706 & $3.1 \mathrm{~N}$ & $1.5 \mathrm{R}$ & $1.9 \mathrm{R}$ & + & - \\
\hline ND 808 & HRSW-ND & ND2857/3/Kitt/Amidon//GR/Stoa SIB & $1.9 \mathrm{R}$ & $2.1 \mathrm{C}$ & $3.9 \mathrm{C}$ & - & + \\
\hline ND 809 & HRSW-ND & ND2831//Parshall/ND706/3/ND721 & $3.4 \mathrm{~N}$ & $1.8 \mathrm{R}$ & $3.9 \mathrm{C}$ & - & + \\
\hline ND 810 & HRSW-ND & ND694/GR*2/RL4137//Amidon & $2.6 \mathrm{~N}$ & $1.6 \mathrm{R}$ & $3.9 \mathrm{C}$ & - & + \\
\hline ND 811 & HRSW-ND & ND724/2*Reeder & $1.9 \mathrm{R}$ & $1.4 \mathrm{R}$ & $3.4 \mathrm{C}$ & - & + \\
\hline NDSW0207 & WW-ND & 9734/AC Vista & $3.2 \mathrm{~N}$ & $2.2 \mathrm{C}$ & $4.1 \mathrm{C}$ & - & + \\
\hline NDSW0217 & WW-ND & Sunstate/9734 & $3.5 \mathrm{~N}$ & $3.0 \mathrm{C}$ & $2.6 \mathrm{C}$ & + & + \\
\hline NDSW0345 & WW-ND & MT9904/Alsen & $3.5 \mathrm{~N}$ & $2.4 \mathrm{C}$ & $4.2 \mathrm{C}$ & + & + \\
\hline NDSW0347 & WW-ND & Reeder//IDO560/9712 & $3.7 \mathrm{~N}$ & $1.8 \mathrm{R}$ & $4.2 \mathrm{C}$ & + & + \\
\hline NDSW0431 & WW-ND & BaiHuo/Kanto107//Express/3/MTHW9420 & $4.0 \mathrm{~N}$ & $2.6 \mathrm{C}$ & $2.2 \mathrm{C}$ & + & + \\
\hline Erik & HRSW-KS & Kitt/2/Waldron/Era & $1.3 \mathrm{R}$ & $1.3 \mathrm{R}$ & $1.5 \mathrm{R}$ & - & - \\
\hline Glenlea & OW-CAN & Pemina*2/Bage/2/CB100 & $4.3 \mathrm{~N}$ & $2.0 \mathrm{R}$ & $1.9 \mathrm{R}$ & + & - \\
\hline $6 \mathrm{~B}-365$ & OW-CAN & Unknown & $1.7 \mathrm{R}$ & $4.1 \mathrm{C}$ & $1.9 \mathrm{R}$ & - & - \\
\hline $6 \mathrm{~B}-662$ & OW-CAN & Unknown & $1.7 \mathrm{R}$ & $1.7 \mathrm{R}$ & $4.0 \mathrm{C}$ & - & + \\
\hline ND 495 & HRSW-ND & Justin*2/3/ND259/Conley//ND112 & $4.5 \mathrm{~N}$ & $2.3 \mathrm{C}$ & $2.5 \mathrm{C}$ & + & + \\
\hline Conway & HRSW-CAN & Chris/Siete-Cerros-66//Neepawa/Opal & $4.0 \mathrm{~N}$ & $1.8 \mathrm{R}$ & $4.1 \mathrm{C}$ & + & + \\
\hline Katepwa & HRSW-CAN & Neepwa*6/RL-2938/3/Neepawa*6//CI-8154/2*Frocor & $4.0 \mathrm{~N}$ & $1.8 \mathrm{R}$ & $4.4 \mathrm{C}$ & + & + \\
\hline Kenyon & HRSW-CAN & Neepawa*5/Buck Manantial & $4.3 \mathrm{~N}$ & $2.4 \mathrm{C}$ & $3.8 \mathrm{C}$ & + & + \\
\hline Coulter & Durum-CAN & DT-188/Dt-224//DT-182 & $4.3 \mathrm{~N}$ & $4.1 \mathrm{~N}$ & $4.3 \mathrm{~N}$ & + & - \\
\hline 4B-160 & OW-CAN & Unknown & $1.7 \mathrm{R}$ & $2.9 \mathrm{~N}$ & $2.5 \mathrm{~N}$ & - & - \\
\hline T. turgidum \#283 & OW-CAN & PI352519 & $1.3 \mathrm{R}$ & $1.4 \mathrm{R}$ & $1.4 \mathrm{R}$ & - & - \\
\hline Intros \#7 & OW-MEX & Unknown & $1.4 \mathrm{R}$ & $1.3 \mathrm{R}$ & $1.3 \mathrm{R}$ & - & - \\
\hline 92 MREHTR28B & OW-CAN & Car853/Coc//Vee'S'/3/E7408/Pam'S'//Hor & $1.6 \mathrm{R}$ & $1.3 \mathrm{R}$ & $1.3 \mathrm{R}$ & - & - \\
\hline 2000 Spelt \#20 & OW-CAN & PI348771/Oberkulm & $1.4 \mathrm{R}$ & $1.2 \mathrm{R}$ & $1.5 \mathrm{R}$ & - & - \\
\hline CIMMYT L \#18 & OW-MEX & Unknown & $1.5 \mathrm{R}$ & $1.3 \mathrm{R}$ & $1.4 \mathrm{R}$ & - & - \\
\hline Saloumini & OW-CAN & Unknown & $1.4 \mathrm{R}$ & $1.4 \mathrm{R}$ & $1.3 \mathrm{R}$ & - & - \\
\hline Mountrail & Durum-ND & D8479/Renville & $2.2 \mathrm{~N}$ & $3.2 \mathrm{~N}$ & $2.2 \mathrm{~N}$ & - & - \\
\hline Ben & Durum-ND & D8024/Monroe & $3.8 \mathrm{~N}$ & $3.6 \mathrm{~N}$ & $2.3 \mathrm{~N}$ & + & - \\
\hline Lebsock & Durum-ND & Munich/D8469 & $2.7 \mathrm{~N}$ & $3.0 \mathrm{~N}$ & $1.9 \mathrm{R}$ & + & - \\
\hline Pierce & Durum-ND & D86117/D88289 & $3.6 \mathrm{~N}$ & $3.2 \mathrm{~N}$ & $2.0 \mathrm{R}$ & + & - \\
\hline Maier & Durum-ND & D8193/D8335 & $3.1 \mathrm{~N}$ & $2.7 \mathrm{~N}$ & $2.5 \mathrm{~N}$ & + & - \\
\hline Plaza & Durum-ND & DT606/D8291 & $3.0 \mathrm{~N}$ & $2.4 \mathrm{~N}$ & $2.7 \mathrm{~N}$ & + & - \\
\hline Dilse & Durum-ND & Maier/D88273 & $3.2 \mathrm{~N}$ & $2.9 \mathrm{~N}$ & $3.3 \mathrm{~N}$ & + & - \\
\hline Grenora & Durum-ND & D901260/D901419 & $2.6 \mathrm{~N}$ & $3.1 \mathrm{~N}$ & $2.5 \mathrm{~N}$ & + & - \\
\hline Divide & Durum-ND & Ben/D901282//Belzer & $2.9 \mathrm{~N}$ & $3.0 \mathrm{~N}$ & $3.0 \mathrm{~N}$ & + & - \\
\hline Alkabo & Durum-ND & D901247/D89263 & $2.1 \mathrm{~N}$ & $2.5 \mathrm{~N}$ & $3.0 \mathrm{~N}$ & + & - \\
\hline $\operatorname{LSD}_{(0.05)}$ & & & 0.5 & 0.6 & 0.5 & & \\
\hline
\end{tabular}


sult, assessment of tan spot resistance on a lesion-type rating scale is preferred $(5,23)$.

Screening wheat seedlings by artificial inoculation in the greenhouse permits examination of large populations for resistance under uniform disease pressure. A positive correlation has been reported between assessment of resistance at seedling stage and rating of adult plants in the field (12). The fact that tan spot in wheat fields occurs in association with other foliar diseases makes it more difficult and error prone to do field screening for tan spot resistance $(20,23)$. Hence, when breeding for tan spot resistance, seedling evaluation of plants under controlled conditions is the preferred methodology.

To date, the fungus $P$. tritici-repentis has eight races. Races 2, 3, and 5 are all reported to have single virulence genes and are reported as the base races, i.e., combination of virulence of races 2,3 , and 5 have resulted in the development of other virulent races (26). Race 1 is the most prevalent race of $P$. tritici-repentis in the northern Great Plains of North America $(2,22,25)$ and is reported to have the virulence of both races 2 and 3 (26). By screening the wheat germ plasm individually with races 2 and 3 , we can predict the expected reaction of a genotype to race 1 . Additionally, races 2 and 3 induce only one disease symptom, i.e., either necrosis or chlorosis in hexaploid and/or tetraploid wheat, thereby making the disease scoring easy, precise, and accurate. Results of this study can be used to predict the reaction of a genotype to other virulent races that are combinations of races 2,3 , and 5 .

All susceptible hexaploid wheat genotypes tested with race 5 developed chlorosis, while durum wheat genotypes exhibited necrosis (Table 1). Upon infiltration of culture filtrate of race 5 to susceptible hexaploid wheat, genotypes showed chlorotic symptoms while susceptible durum exhibited insensitive reaction, indicating culture filtrate lacks the factor present in the spore suspension, which causes necrosis. These results indicate race 5 isolates may possibly have at least two disease inducing factors, one causing necrosis in durum and the other, toxin Ptr ToxB, inducing chlorosis in hexaploid wheat. Similar views have been suggested by Gamba and Lamari (18) and Singh and Hughes (32).

Our results indicate that the most genotypes are resistant to $P$. tritici-repentis race 3 followed by race 5 and race 2 (Table 1). In recent years, race 5 isolates have increased in prevalence among the $P$. tritici-repentis population, with a single isolate found in the ManitobaSaskatchewan border (25) and a significantly higher proportion of this race detected in North Dakota (2). Most wheat breeding programs in the northern Great Plains use race 1 isolates and natural inoculum as a means of screening for tan spot resistance $(5,8)$. Although race 1 isolates induce both necrosis and chlorosis, similar to the combined symptoms induced by races 2 and 3, they do not have the virulence of race 5 . Hence, we may not be able to eliminate susceptibility for race 5 in tan spot resistance breeding. It is recommended that future tan spot screening should include multiple race screening or a mixture of races 1 and 5 to develop tan spot resistant varieties.

\section{ACKNOWLEDGMENTS}

Financial support from Wheat Research and Promotion Council, Minnesota, USA, North Dakota Wheat Commission, and State Board of Agricultural Research and Education, North Dakota, USA, is gratefully acknowledged. Technical help provided by Seema Singh is greatly appreciated.

\section{LITERATURE CITED}

1. Ali, S., and Francl, L. J. 2002. A new race of Pyrenophora tritici-repentis from Brazil. Plant Dis. 86:1050.

2. Ali, S., and Francl, L. J. 2003. Population race structure of Pyrenophora tritici-repentis prevalent on wheat and noncereal grasses in the Great Plains. Plant Dis. 87:418-422.

3. Ali, S., Ling, H., Meinhardt, S., and Francl, L. 2002. A new race of Pyrenophora triticirepentis that produces a putative host-selective toxin. (Abstr.) Phytopathology 92:S3.

4. Anderson, J. A., Effertz, R. J., Faris, J. D., Francl, L. J., Meinhardt, S. W., and Gill, B. S. 1999. Genetic analysis of sensitivity to a Pyrenophora tritici-repentis necrosis-inducing toxin in durum and common wheat. Phytopathology 89:293-297.

5. Brûlé-Babel, A. L., and Lamari, L. 1992. Evaluation of field screening techniques for tan spot resistance in spring wheat. Pages 3943 in: Advances in tan spot research: Proc. 2nd Int. Tan Spot Workshop. L. J. Francl, J. M. Krupinsky, and M. P. McMullen, eds. North Dakota State University, Fargo.

6. Ciuffetti, L. M., and Tuori, R. P. 1999. Advances in the characterization of the Pyrenophora tritici-repentis-wheat interaction. Phytopathology 89:444-449.

7. De Wolf, E. D., Effertz, R. J., Ali, S., and Francl, L. J. 1998. Vistas of tan spot research. Can. J. Plant Pathol. 20:349-370.

8. Duguid, S. D., and Brûlé-Babel, A. L. 2001. Inheritance of resistance to a necrosis- and chlorosis-inducing isolate from race 1 of Pyrenophora tritici-repentis in spring wheat. Can. J. Plant Sci. 81:519-525.

9. Duguid, S. D., and Brûlé-Babel, A. L. 2001. Inheritance and interaction of spring wheat (Triticum aestivum L.) resistance to race 2 and race 3 of Pyrenophora tritici-repentis (Died.) Drechs. Can. J. Plant Sci. 81:527-533.

10. Effertz, R. J., Meinhardt, S. W., Anderson, J. A., Jordahl, J. G., and Francl, L. J. 2002. Identification of a chlorosis-inducing toxin from Pyrenophora tritici-repentis and the chromosomal location of an insensitivity locus in wheat. Phytopathology 92:527-533.

11. Elias, E., Cantrell, R. G., and Horsford, R. M., Jr. 1989. Heritability of resistance to tan spot in durum wheat and its association with other agronomic traits. Crop Sci. 29:299-304.

12. Evans, C. K., Hunger, R. M., and Siegerist, W. C. 1999. Comparison of greenhouse and field testing to identify wheat resistant to tan spot. Plant Dis. 83:269-273.

13. Faris, J. D., Anderson, J. A., Francl, L. J., and Jordahl, J. G. 1996. Chromosomal location of a gene conditioning insensitivity in wheat to a necrosis-inducing culture filtrate from Pyrenophora tritici-repentis. Phytopathology 86:459463.

14. Faris, J. D., Anderson, J. A., Francl, L. J., and Jordahl, J. G. 1997. RFLP mapping of resistance to chlorosis induction by Pyrenophora tritici-repentis. Theor. Appl. Genet. 94:98103.

15. Friesen, T. L., Ali, S., Kianian, S., Francl, L. J., and Rasmussen, J. B. 2003. Role of host sensitivity to Ptr ToxA in development of tan spot of wheat. Phytopathology 93:397-401.

16. Friesen, T. L., and Faris, J. D. 2004. Molecular mapping of resistance to Pyrenophora triticirepentis race 5 and sensitivity to Ptr ToxB in wheat. Theor. Appl. Genet. 109:464-471.

17. Friesen, T. L., Rasmussen, J. B., Kwon, C. Y., Ali, S., Francl, L. J., and Meinhardt, S. W. 2002. Reaction of Ptr ToxA-insensitive wheat mutants to Pyrenophora tritici-repentis race 1. Phytopathology 92:38-42.

18. Gamba, F. M., and Lamari, L. 1998. Mendelian inheritance of resistance to tan spot (Pyrenophora tritici-repentis) in selected genotypes of durum wheat (Triticum turgidum) Can. J. Plant Pathol. 20:408-414.

19. Gamba, F. M., Lamari, L., and Brûlé-Babel, A. 1998. Inheritance of race-specific necrotic and chlorotic reactions induced by Pyrenophora tritici-repentis in hexaploid wheats. Can. J. Plant Pathol. 20:401-407.

20. Gilbert, J., and Woods, S. M. 2001. Leaf spot diseases of spring wheat in southern Manitoba farm fields under conventional and conservation tillage. Can. J. Plant Sci. 81:551-559.

21. Lamari, L., and Bernier, C. C. 1989. Toxin of Pyrenophora tritici-repentis: Host specificity, significance in disease, and inheritance of host reaction. Phytopathology 79:740-744.

22. Lamari, L., and Bernier, C. C. 1989. Virulence of isolates of Pyrenophora tritici-repentis on 11 wheat cultivars and cytology of differential host reactions. Can. J. Plant Pathol. 11:284290.

23. Lamari, L., and Bernier, C. C. 1989. Evaluation of wheat reaction to tan spot (Pyrenophora tritici-repentis) based on lesion type Can. J. Plant Pathol. 11:49-56.

24. Lamari, L., and Bernier, C. C. 1991. Genetics of $\tan$ necrosis and extensive chlorosis in tan spot of wheat caused by Pyrenophora triticirepentis. Phytopathology 81:1092-1095.

25. Lamari, L., Gilbert, J., and Tekauz, A. 1998. Race differentiation in Pyrenophora triticirepentis and surveys of physiologic variation in western Canada. Can. J. Plant Pathol. 20:396-400.

26. Lamari, L., Strelkov, S. E., Yahyaoui, A., Orabi, J., and Smith, R. B. 2003. The identification of two new races of Pyrenophora triticirepentis from the host center of diversity confirms a one-to-one relationship in tan spot of wheat. Phytopathology 93:391-396.

27. Manning, V. A., Pandelova, I., and Ciuffetti, L M. 2002. A race for a novel host-selective toxin. (Abstr.) Phytopathology 92:S51.

28. McMullen, M. 2002. Prevalence and severity of tan spot and the Septoria leaf disease complex on wheat and relationship to previous crop, North Dakota 1998-2001. Pages 1-3 in: Proc. 4th Int. Wheat Tan Spot Spot Blotch Workshop. J. B. Rasmussen, T. L. Friesen, and S. Ali, eds. North Dakota State University, Fargo.

29. Orolaza, N. P., Lamari, L., and Ballance, G. M. 1995. Evidence of a host-specific chlorosis toxin from Pyrenophora tritici-repentis, the causal agent of tan spot of wheat. Phytopathology 85:1282-1287.

30. Riede, C. R., Francl, L. J., Anderson, J. A., Jordahl, J. G., and Meinhardt, S. W. 1996. Additional sources of resistance to tan spot of wheat. Crop Sci. 36:771-777.

31. SAS Institute. 1999. SAS/STAT User's Guide, 
Rel. 8.2, 8.1, 8.0. SAS Institute, Cary, NC.

32. Singh, P. K., and Hughes, G. R. 2004. Genetic analysis of differential response of Pyrenophora tritici-repentis, race 5 on durum and common wheat. (Abstr.) Can. J. Plant Pathol. $26: 423$.

33. Singh, P. K., and Hughes, G. R. 2005. Genetic control of resistance to tan necrosis induced by
Pyrenophora tritici-repentis, races 1 and 2, in spring and winter wheat genotypes. Phytopathology 95:172-177.

34. Singh, P. K., and Hughes, G. R. 2006. Genetic similarity among isolates of Pyrenophora tritici-repentis, causal agent of tan spot of wheat. J. Phytopathol. 154:178-184.

35. Tomas, A., and Bockus, W. W. 1987. Cultivar specific toxicity of culture filtrate of Pyrenophora tritici-repentis. Phytopathology 77:13371366.

36. Zhang, H. F., Francl, L. J., Jordahl, J. G., and Meinhardt, S. W. 1997. Structural and physical properties of a necrosis-inducing toxin from Pyrenophora tritici-repentis. Phytopathology 87:154-160. 